\title{
Баллистический магнетотранспорт в подвешенном двумерном электронном газе с периодической решеткой антиточек
}

\author{
() Е.Ю. Жданов ${ }^{1,2}$, А.Г. Погосов ${ }^{1,2}$, М.В. Буданцев ${ }^{1}$, Д.А. Похабов ${ }^{1,2}$, А.К. Бакаров ${ }^{1,2}$ \\ ${ }^{1}$ Институт фризики полупроводников Сибирского отделения Российской академии наук, \\ 630090 Новосибирск, Россия \\ ${ }^{2}$ Новосибирский государственный университет, \\ 630090 Новосибирск, Россия \\ E-mail: zhdanov@isp.nsc.ru
}

(Получена 5 апреля 2016 г. Принята к печати 12 апреля 2016 г.)

\begin{abstract}
Исследовано магнетосопротивление подвешенных полупроводниковых наноструктур с двумерным электронным газом, структурированным периодическими квадратными решетками антиточек. Показано, что баллистический режим электронного транспорта сохраняется после отрыва образцов от подложки. Проведен прямой сравнительный анализ соизмеримых осцилляций магнетосопротивления, а также их температурных зависимостей в образцах до и после подвешивания. Обнаружено, что температурные зависимости практически идентичны для неподвешенных и подвешенных образцов, в то время как в нелинейном режиме, обусловленном пропусканием постоянного тока, наблюдаются существенные отличия. Соизмеримые осцилляции в подвешенных образцах оказываются более устойчивыми по отношению к воздействию постоянного тока, что предположительно может быть объяснено усилением электрон-электронного взаимодействия после отрыва наноструктур от высокодиэлектрической подложки.
\end{abstract}

DOI: 10.21883/FTP.2017.01.43988.8259

\section{1. Введение}

Оторванные от подложки (или „подвешенные“) наноструктуры с высокоподвижным двумерным электронным газом (ДЭГ) являются примером так называемых наноэлектромеханических систем. Такие системы интересны с точки зрения практических применений, что в основном обусловлено наличием в них сцепки электрических и механических степеней свободы [1]. Однако даже неколеблющиеся подвешенные наноструктуры вызывают интерес исследователей благодаря ряду их уникальных свойств, среди которых можно отметить: усиленное электрон-электронное взаимодействие [2], отсутствие отвода тепла непосредственно через подложку и связанные с этим особенности явлений теплопереноса $[3,4]$. Несмотря на это, фундаментальные вопросы электронного транспорта, как квантового, так и классического, остаются мало изученными в таких системах.

В настоящей статье описываются результаты исследований электронного транспорта в баллистическом режиме в подвешенном ДЭГ, структурированном периодическими решетками искусственных рассеивателей антиточек. Мезоскопические явления в обычных неподвешенных структурах с решетками антиточек исследуются достаточно давно и подробно, и на настоящий момент такие структуры являются хорошо изученными. Они представляют собой один из примеров реализации твердотельных электронных биллиардов Синая [5]. Решетки антиточек - это системы с динамическим хаосом, в фазовом пространстве которых присутствуют области, отвечающие регулярному движению по устойчивым электронным траекториям. Устойчивые электрон- ные траектории, возникающие в определенных магнитных полях, обусловливают множество интересных особенностей явлений электронного транспорта. Например, в магнетосопротивлении решеток антиточек проявляются так называемые соизмеримые осцилляции, которые обусловлены совпадением в определенных магнитных полях размеров решетки и циклотронного диаметра орбиты электронов - возникновением геометрических резонансов [6]. Кроме того, обнаружен аномальный рост нелокального сопротивления [7] в магнитных полях, при которых выполняются условия соизмеримости, изучены магнетополевые осцилляции диффузионной термоэдс, имеющие ту же природу, что и осцилляции магнетосопротивления [8]. Исследованы также нелинейные эффекты при пропускании большого постоянного тока и показано, что влияние постоянного электрического поля не сводится только к эффектам разогрева, а приводит также к разрушению устойчивых электронных траекторий за счет дрейфа в скрещенных электрическом и магнитном полях [9]. Помимо перечисленных явлений, в периодических решетках антиточек обнаружены квантовые интерференционные явления, проявляющиеся в таких эффектах, как эффект Ааронова-Бома [10], эффект слабой локализации [11], мезоскопические флуктуации кондактанса [12], термоэдс [13] и др. Все эти явления изучались в неподвешенных электронных системах, т.е. в системах, находящихся в массиве полупроводника. Данная статья посвящена исследованию баллистического электронного транспорта в решетках антиточек, оторванных от подложки. Особое внимание уделено исследованию соизмеримых осцилляций магнетосопротивления в нелинейном режиме. 


\section{2. Методика эксперимента}

Экспериментальные образцы изготавливались на основе гетероструктур AlAs/GaAs с ДЭГ, выращенных методом молекулярно-лучевой эпитаксии. ДЭГ характеризуется высокой подвижностью электронов $\mu=$ $=2 \cdot 10^{6} \mathrm{~cm}^{2} /(\mathrm{B} \cdot \mathrm{c})$ при концентрации $n=7 \cdot 10^{11} \mathrm{~cm}^{-2}$. Гетероструктуры толщиной 160 нм выращивались поверх жертвенного слоя $\mathrm{Al}_{0.8} \mathrm{Ga}_{0.2} \mathrm{As}$ толщиной $400 \mathrm{Hм}$, который впоследствии удалялся из-под них при помощи изотропного селективного травления в $2 \%$-ном водном растворе плавиковой кислоты. Полученные образцы представляли собой холловские мостики, латеральные размеры которых задавались при помощи оптической литографии. Длина подвешенной части холловских мостиков составляла 15 мкм, а ширина 3 мкм. После отрыва мостиков от подложки концентрация ДЭГ практически не менялась, а подвижность, измеренная при температуре $4.2 \mathrm{~K}$, уменьшалась не более чем в 2 раза. При помощи электронной литографии с последующим анизотропным плазмохимическим травлением изготовленные мостики вместе с потенциометрическими контактами покрывались квадратной периодической решеткой антиточек (см. вставку на рис. 1). Были изготовлены серии образцов, отличающиеся периодом решетки (от 500 до 800 нм) и диаметром антиточек (от 100 до 200 нм). Измерения магнетосопротивления полученных мостиков проводились методом синхронного детектирования на частоте 7 Гц в режиме линейного отклика, величина тянущего тока составляла 0.1 мкА. Измерения проводились в широком температурном диапазоне от $480 \mathrm{MK}$ до $50 \mathrm{~K}$. Для изучения нелинейных эффектов помимо тянущего переменного тока через образец пропускался постоянный ток (до 100 мкА). Все экспериментальные результаты, приведенные далее, получены на образцах с квадратной решеткой антиточек с периодом решетки $d=600$ нм и диаметром антиточек $2 a=200$ нм. Результаты хорошо воспроизводятся для серии образцов с теми же параметрами решетки, а характерные особенности магнетосопротивления полностью сохраняются и в образцах с другими параметрами решеток.

\section{3. Экспериментальные результаты}

На рис. 1 представлены зависимости продольного и холловского магнетосопротивления подвешенного баллистического мостика с решеткой антиточек. Продольное магнетосопротивление демонстрирует два пика соизмеримых осцилляций в магнитных полях 0.18 и 0.48 Тл. В холловском магнетосопротивлении в этих же магнитных полях также видны особенности. Эти особенности имеют баллистическую природу (этот факт подтверждается, в частности, отсутствием температурной зависимости этих особенностей при уменьшении температуры) и обусловлены геометрическими резонансами.

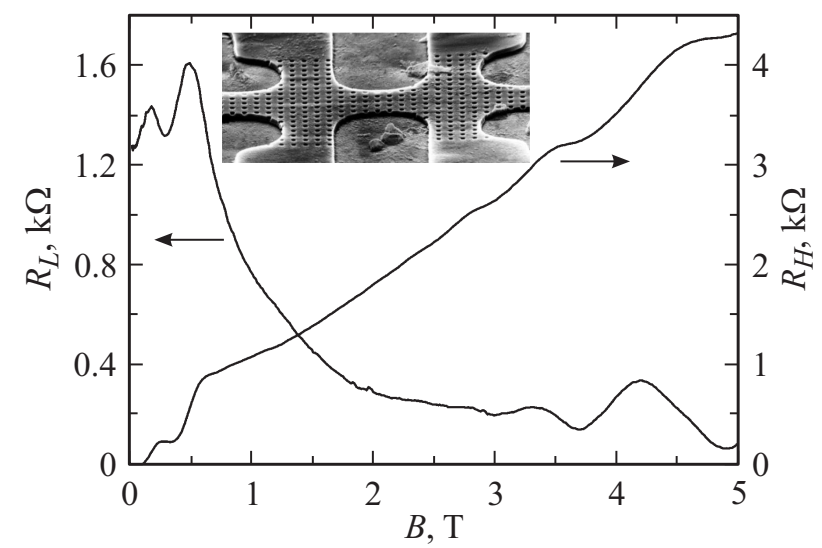

Рис. 1. Зависимость продольного и холловского сопротивления подвешенного холловского мостика с решеткой антиточек (период решетки антиточек $d=600 \mathrm{HM}$, литографический диаметр антиточек $2 a=200 \mathrm{HM})$. На вставке - изображение подвешенного мостика с решеткой антиточек с периодом $d=600$ нм и диаметром антиточек $2 a=200$ нм. Изображение получено методом сканирующей электронной микроскопии при падении электронного пучка под углом $60^{\circ}$ относительно нормали к поверхности образца.

В сильных магнитных полях (более 2 Тл) наблюдаются осцилляции Шубникова-де-Гааза. Аналогичное поведение магнетосопротивления наблюдается и на неподвешенных образцах. Условие соизмеримости для главного максимума (при $B=0.48$ Тл) определяется равенством периода $d$ решетки антиточек циклотронному диаметру электрона [14]: $d=2 r_{\mathrm{c}}$, где

$$
r_{\mathrm{c}}=\frac{h}{e B} \sqrt{\frac{n}{2 \pi}}=\frac{\sqrt{2 E_{\mathrm{F}} m^{*}}}{e B} .
$$

Здесь $h-$ постоянная Планка, $e-$ заряд электрона, $B$ - величина магнитного поля, $n-$ концентрация электронов (определялась из периода осцилляций Шубникова-де-Гааза), $m^{*}$ - эффективная масса электронов в $\mathrm{GaAs}$, а $E_{\mathrm{F}}-$ энергия Ферми. В магнитном поле $B=0.48$ Тл циклотронный диаметр $2 r_{\mathrm{c}} \approx 600 \mathrm{Hм}$, что совпадает с периодом решетки антиточек. Наличие соизмеримых осцилляций, имеющих баллистическую природу, в магнетосопротивлении подвешенных образцов говорит о сохранении высокой подвижности и большой длине свободного пробега электронов в подвешенном ДЭГ (для возникновения наблюдаемых пиков должны сформироваться устойчивые траектории электронов, совершающих несколько столкновений с антиточками до рассеяния на заряженной примеси).

На рис. 2, $a$ и $b$ представлены температурные зависимости соизмеримых осцилляций магнетосопротивления для неподвешенного и подвешенного образцов. Эти зависимости схожи: в обоих случаях амплитуда соизмеримых осцилляций убывает с ростом температуры, и полное подавление осцилляций происходит при одинаковой тем- 

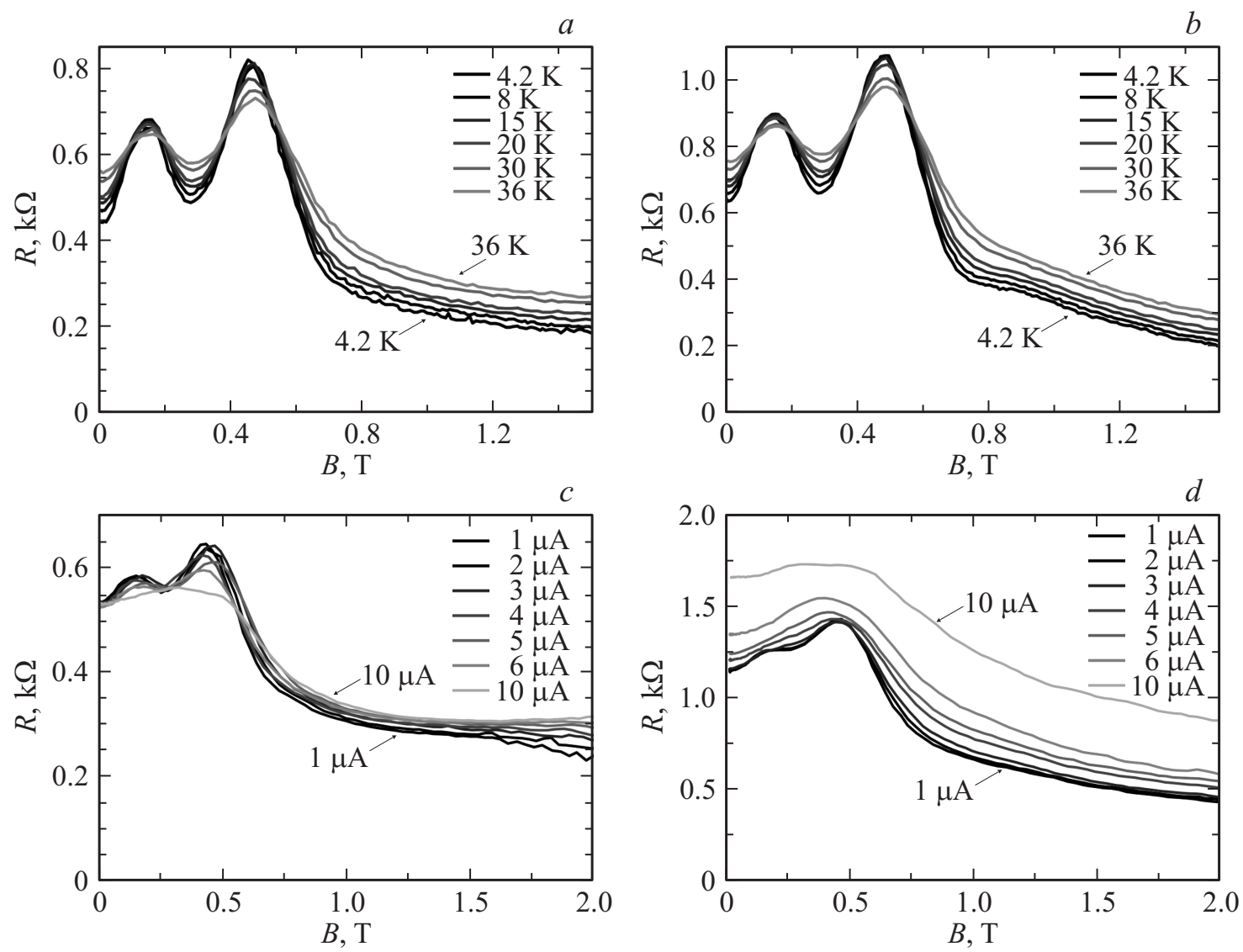

Рис. 2. Зависимость продольного магнетосопротивления неподвешенного $(a)$ и подвешенного $(b)$ холловских мостиков с решеткой антиточек от температуры, а также неподвешенного $(c)$ и подвешенного $(d)$ холловских мостиков с решеткой антиточек от величины пропускаемого тянущего тока (период решетки антиточек $d=600$ нм, литографический диаметр антиточек $2 a=200$ нм).

пературе (около $50 \mathrm{~K})$. В свою очередь осцилляции Шубникова-де-Гааза (не показаны на рис. 2), обусловленные квантованием уровней Ландау, подавляются при меньших температурах (около $10 \mathrm{~K}$ ).

Подавление соизмеримых осцилляций при повышении температуры связано с размытием циклотронного радиуса за счет теплового размытия энергии Ферми. При температуре порядка $50 \mathrm{~K}(\approx 4$ мэВ $)$ тепловое размытие энергии Ферми $\left(E_{\mathrm{F}} \approx 20\right.$ мэВ при концентрации ДЭГ $n=7 \cdot 10^{11} \mathrm{~cm}^{-2}$ ) составляет $20 \%$. Это, согласно (1), приводит к размытию циклотронного радиуса на $10 \%$. Как видно из рис. 2 , ширина основного пика осцилляций $(B=0.48$ Тл) составляет $\Delta B \approx 0.1$ Тл, что соответствует такому же разбросу циклотронного радиуса $\Delta r_{\mathrm{c}} / r_{\mathrm{c}} \approx 10 \%$. Приведенная оценка показывает, что разогрев может являться главной причиной подавления соизмеримых осцилляций. Причем, как видно из рис. 2, $a$ и $b$, это подавление происходит одинаково как в неподвешенных, так и в подвешенных образцах.

Однако в нелинейном режиме поведение неподвешенных и подвешенных образцов существенно отличается. Для изучения магнетосопротивления в нелинейном режиме через токовые контакты структуры вме- сте с малым переменным током пропускался большой (до 100 мкА) постоянный ток. Было обнаружено, что при увеличении амплитуды пропускаемого постоянного тока как в подвешенных, так и в неподвешенных образцах происходит подавление соизмеримых осцилляций, однако характер подавления сильно отличается (см. рис. 2, $c$ и $d$ ).

Можно выделить две основные причины, ответственные за подавление соизмеримых осцилляций в структурах с решетками антиточек при пропускании постоянного тока. Первая связана с эффектами разогрева, а вторая - c разрушением устойчивых траекторий за счет дрейфа электронов в скрещенных электрическом и магнитном полях [9]. В первом случае амплитуда подавления осцилляций должна определяться величиной мощности, подводимой к образцу, а во втором величиной электрического поля. Рассмотрим влияние каждого из этих эффектов подробнее, приняв в качестве удобной величины для сравнения эффектов подавления соизмеримых осцилляций величину относительного изменения амплитуды пиков $\delta R / R_{0}$, где $\delta R-$ разница между сопротивлением в магнитном поле, соответствующем пику осцилляций, и сопротивлением $R_{0}$ в нулевом магнитном поле. 

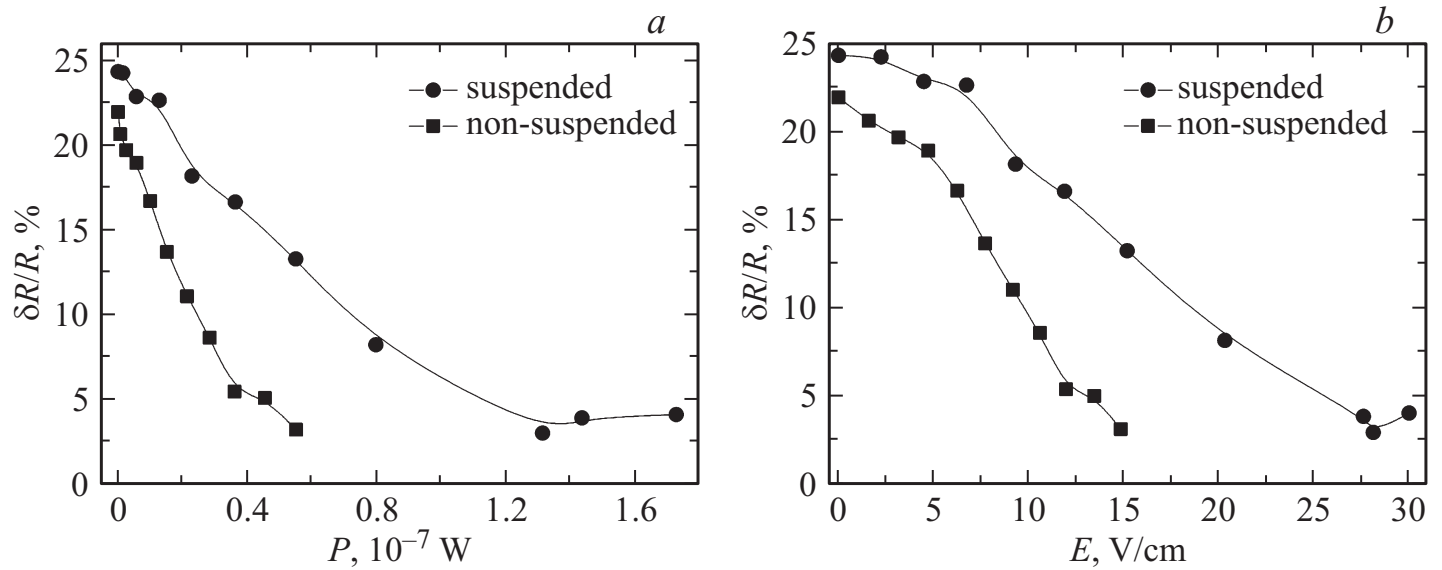

Рис. 3. Зависимость относительной амплитуды главного пика соизмеримых осцилляций от мощности $(a)$, выделяющейся в образце, и от величины приложенного электрического поля $(b)$ для подвешенных и неподвешенных образцов.

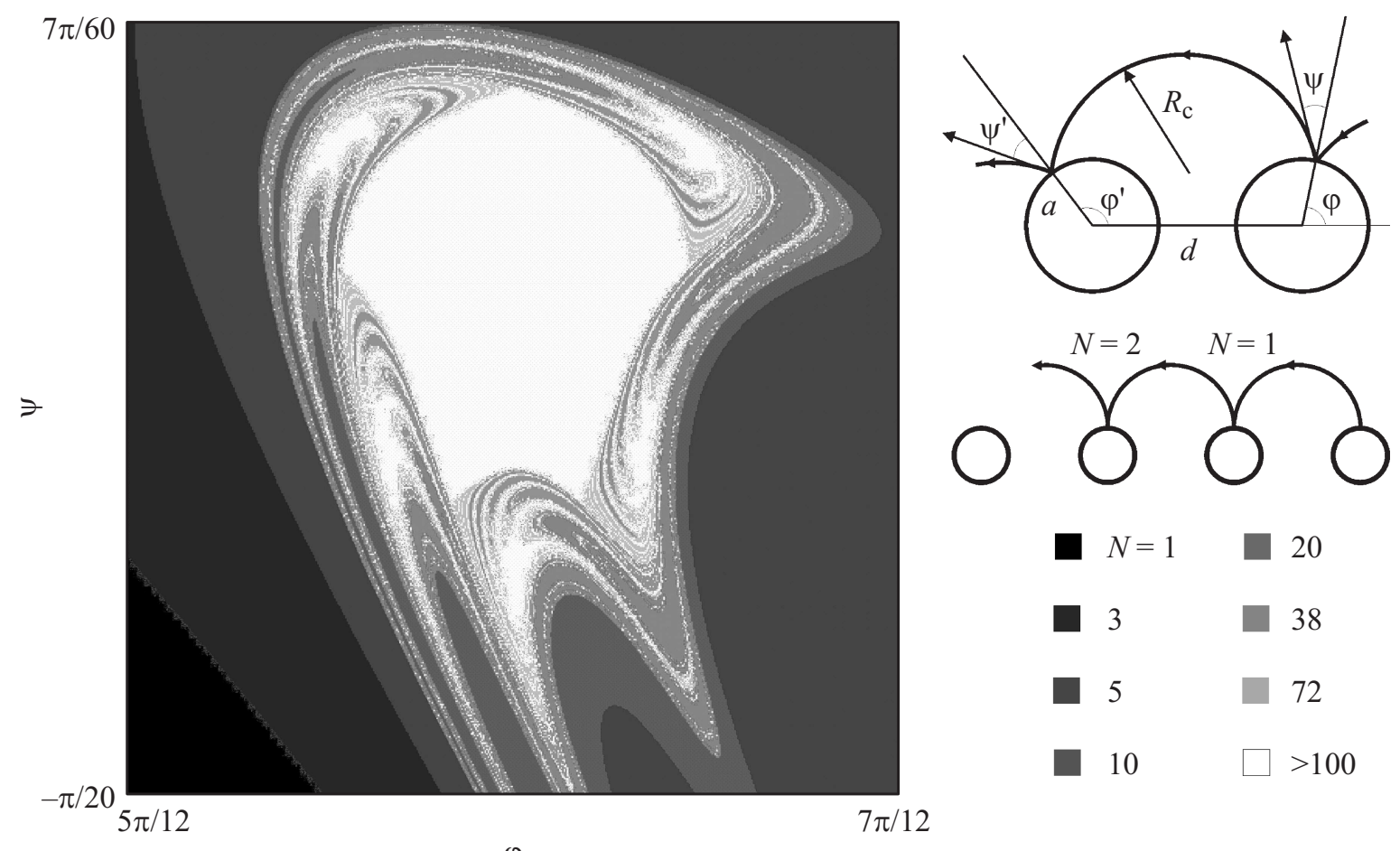

$\varphi$

Рис. 4. Рассчитанные области устойчивости убегающих траекторий, „скачущих“ вдоль ряда антиточек, в угловых координатах, где $\varphi$ характеризует угловое положение электрона при столкновении с антиточкой, а $\psi-$ угол отражения электрона от антиточки. Градацией серого показано количество $N$ последовательных скачков, происходящих без сбоя.

\section{1. Эфффекты разогрева}

При подавлении амплитуды соизмеримых осцилляций током, например вдвое, осцилляции Шубникова-де-Гааза остаются еще не полностью подавленными. Следовательно, в этом случае температура электронной системы меньше температуры подавления осцилляций Шубникова-де-Гааза, которая равна, как было сказано выше, $10 \mathrm{~K}$, и, следовательно, температура системы значительно ниже температуры подавления соиз- меримых осцилляций $(50 \mathrm{~K})$. Это означает, что эффекты разогрева имеют место, однако их вклад в подавление соизмеримых осцилляций не является определяющим. Для сравнения влияния эффектов разогрева на подавление соизмеримых осцилляций для подвешенных и неподвешенных образцов использовалась зависимость относительной амплитуды главного пика соизмеримых осцилляций $\delta R / R_{0}$ от подводимой к образцу мощности (рис. $3, a$ ), которая определялась величинами пропускаемого тока и измеренного сопротивления $\left(P=I^{2} R\right)$. 
Из рис. 3, $a$ видно, что соизмеримые осцилляции магнетосопротивления в подвешенных образцах подавляются при большей подводимой мощности, чем в неподвешенных, несмотря на идентичность температурных зависимостей этих осцилляций. Более того, такое поведение обратно наблюдаемому при изучении пробоя квантового эффекта Холла [3], где в подвешенных образцах зануления продольного магнетосопротивления исчезали при меньших критических греющих токах, чем в неподвешенных образцах. Таким образом, необычное поведение магнетосопротивления в подвешенных образцах нельзя объяснить эффектами разогрева.

\section{2. Дрейф электронной орбиты}

Оценим величину дрейфового смещения центра орбиты электрона между двумя столкновениями с антиточками. В электрическом поле, при котором происходит практически полное подавление соизмеримых осцилляций $(E \approx 10 \mathrm{~B} / \mathrm{cm}$, значение рассчитано как отношение падения напряжения на образце к его размеру), эта величина оказывается равной $l=\pi v_{d} / \omega_{\mathrm{c}} \approx 5 \mathrm{Hм}$ (где $v_{d}=E / B-$ скорость дрейфа). Эта величина существенно меньше, чем размер антиточек $(a=200 \mathrm{Hм})$, однако ее необходимо сравнивать с размером области устойчивости, который также меньше, чем размер антиточки. Действительно, на рис. 4 показаны рассчитанные области устойчивости траекторий, убегающих вдоль ряда квадратной решетки антиточек, в угловых координатах $\varphi$ (характеризует угловое положение электрона при столкновении с антиточкой) и $\psi$ (характеризует угол отражения электрона от антиточки). Так как в немодулированном ДЭГ длина свободного пробега электронов конечна и составляет примерно 5 мкм, то траектории, которые вносят существенный вклад в сопротивление, совершают около 10 скачков вдоль ряда антиточек до рассеяния на случайной примеси. Из рис. 4 видно, что для этого случая размер области устойчивости составляет $\Delta \varphi \approx \pi / 12$. За один скачок за счет дрейфа может происходить отклонение $\Delta \varphi \approx 2 l / a \approx \pi / 60$, которое будет расти степенным образом с каждым последующим скачком и может превысить размер области устойчивости. Приведенная оценка показывает, что дрейф в скрещенных полях может вносить существенный вклад в подавление соизмеримых осцилляций, причем очевидно, что влияние дрейфа в одном и том же магнитном поле определяется величиной приложенного электрического поля.

Для сравнения влияния этого эффекта в подвешенных и неподвешенных образцах была построена зависимость относительной амплитуды соизмеримых осцилляций магнетосопротивления $\delta R / R_{0}$ от величины приложенного электрического поля (рис. $3, b)$. Из рис. $3, b$ видно, что, подобно эффектам разогрева, соизмеримые осцилляции в подвешенных образцах оказываются более устойчивыми к внешнему воздействию, чем в неподвешенных образцах.

\section{4. Обсуждение результатов}

Таким образом, в нелинейном режиме при пропускании постоянного тока через образец возникают эффекты разогрева и дрейф в скрещенных полях, что действительно может приводить к подавлению соизмеримых осцилляций магнетосопротивления. Однако этими причинами невозможно объяснить различия в поведении этих осцилляций в подвешенных и неподвешенных образцах: дрейф в скрещенных полях должен давать одинаковый вклад, а эффекты разогрева, в силу ослабленного теплоотвода, должны вносить даже больший вклад в подавление соизмеримых осцилляций в подвешенных образцах. Это означает, что существует иная причина, определяющая подавление соизмеримых осцилляций постоянным током.

Такой причиной может являться существенное неравновесие функции распределения электронов в нелинейном режиме, возникающее при пропускании постоянного тока большой амплитуды. Тогда различие в поведении подавления соизмеримых осцилляций может быть объяснено разным временем термализации электронов в неподвешенных и подвешенных образцах. Так как после отрыва структур от подложки происходит усиление электрон-электронного взаимодействия за счет того, что силовые линии электрического поля „зажимаются“ внутри тонкой подвешенной части образца с высокой диэлектрической проницаемостью [2], то это приводит к уменьшению времени термализации электронов в подвешенных структурах. Следовательно, при увеличении амплитуды постоянного тянущего тока функция распределения электронов в неподвешенном образце становится существенно неравновесной, в то время как в подвешенном образце она еще остается близкой к равновесной при той же величине постоянного тока, и соизмеримые осцилляции в подвешенных образцах подавляются не полностью. Более детальное объяснение экспериментально наблюдаемого поведения магнетосопротивления требует дальнейших теоретических исследований.

\section{5. Заключение}

Обнаружены соизмеримые осцилляции магнетосопротивления в полупроводниковых структурах с двумерным электронным газом, структурированным периодическими квадратными решетками антиточек, которые сохраняются и после подвешивания образцов. Проведен прямой сравнительный анализ соизмеримых осцилляций, а также их температурных зависимостей в образцах до и после подвешивания. Обнаружено, что температурные зависимости соизмеримых осцилляций практически идентичны для подвешенных и неподвешенных образцов, в то время как в нелинейном режиме, возникающем при пропускании постоянного тока, наблюдаются существенные отличия. Соизмеримые осцилляции магнетосо- 
противления в подвешенных образцах оказались более устойчивыми по отношению к воздействию постоянного электрического тока, что предположительно может быть объяснено усилением электрон-электронного взаимодействия после подвешивания наноструктур.

Работа выполнена при поддержке РФФИ (№ 16-02-00579).

\section{Список литературы}

[1] K.L. Ekinci, M.L. Roukes. Rev. Sci. Instrum., 76, 061101 (2005).

[2] A.A. Shevyrin, A.G. Pogosov, M.V. Budantsev, A.K. Bakarov, A.I. Toropov, S.V. Ishutkin, E.V. Shesterikov. Appl. Phys. Lett., 104, 203102 (2014).

[3] A.G. Pogosov, M.V. Budantsev, E.Yu. Zhdanov, D.A. Pokhabov, A.K. Bakarov, A.I. Toropov. Appl. Phys. Lett., 100, 181902 (2012).

[4] E.Yu. Zhdanov, A.G. Pogosov, M.V. Budantsev, D.A. Pokhabov. AIP Conf. Proc., 1566, 211 (2013).

[5] Я.Г. Синай. УМН, 25, 141 (1970).

[6] Э.М. Баскин, Г.М. Гусев, З.Д. Квон, А.Г. Погосов, М.В. Энтин. Письма ЖЭТФ, 55, 649 (1992).

[7] М.В. Буданцев, З.Д. Квон, А.Г. Погосов, А.Е. Плотников, Н.Т. Мошегов, А.И. Торопов. Письма ЖЭТФ, 63, 336 (1996).

[8] A.G. Pogosov, M.V. Budantsev, D. Uzur, A. Nogaret, A.E. Plotnikov, A.K. Bakarov, A.I. Toropov. Phys. Rev. B, 66, 201303 (2002).

[9] Г.М. Гусев, 3.Д. Квон, А.Г. Погосов, М.М. Воронин. Письма ЖЭТФ, 65, 237 (1997).

[10] Г.М. Гусев, З.Д. Квон, Л.В. Литвин, Ю.В. Настаушев, А.К. Калагин, А.И. Торопов. Письма ЖЭТФ, 55, 129 (1992).

[11] Г.М. Гусев, 3.Д. Квон, А.Г. Погосов, П. Басмаджи. ЖЭТФ, 110, 696 (1996).

[12] М.В. Буданцев, З.Д. Квон, А.Г. Погосов, Л.В. Литвин, В.Г. Мансуров, В.П. Мигаль, С.П. Мощенко, Ю.В. Настаушев. Письма ЖЭТФ, 59, 614 (1994).

[13] М.В. Буданцев, Р.А. Лавров, А.Г. Погосов, А.Е. Плотников, А.К. Бакаров, А.И. Торопов, Д.К. Мод, Ж.К. Порталь. Письма ЖЭТФ, 79, 201 (2004).

[14] D. Weiss, K.V. Klitzing, K. Ploog, G. Weimann. Europhys. Lett., 8, 179 (1989).

Редактор А.Н. Смирнов

\section{Ballistic magnetotransport in suspended two dimensional electron gas with periodical lattices of antidots}

\author{
E.Yu. Zhdanov ${ }^{1,2}$, A.G. Pogosov 1,2, M.V. Budantsev ${ }^{1}$, \\ D.A. Pokhabov 1,2, A.K. Bakarov ${ }^{1,2}$ \\ ${ }^{1}$ Institute of Semiconductor Physics, \\ Siberian Branch, Russian Academy of Sciences, \\ 630090 Novosibirsk, Russia \\ ${ }^{2}$ Novosibirsk State University, \\ 630090 Novosibirsk, Russia
}

\begin{abstract}
Ballistic electron transport in semiconductor heterostructures containing two dimensional electron gas structured with periodical square lattices of antidots has been studied. Preservation of the ballistic regime of electron transport after suspension has been demonstrated. Direct comparative analysis of commensurability oscillations of magnetoresistance and its temperature dependences in samples before and after the suspension has been carried out. We found that temperature dependences are almost identical for suspended and non-suspended samples while nonlinear effects caused by passing $d c$-current essentially differ. Commensurability oscillations in suspended samples have been more stable with respect to the influence of $d c$-current than oscillations in non-suspended samples. This fact can be explained by strengthening of electron-electron interaction after the detachment of samples from the substrate with large dielectric constant.
\end{abstract}

